\title{
Aggregatibacter actinomycetemcomitans en enfermedad periodontal crónica.
}

Aggregatibacter actinomycetemcomi-

tans in chronic periodontal disease.

Fecha de Recepción

06 de septiembre de 2012
Aggregatibacter actinomycetemcomi-

tans na doença periodontal crônica.
Profesora Adjunta. Cátedra de Microbiología e Inmunología. Facultad de Odontología.

\section{Cynthya Sin}

Auxiliar docente de primera categoría. Cátedra de Microbiología e Inmunología. Becaria de Secretaría General de Ciencia y Técnica.

\section{Resumen}

La enfermedad periodontal se asocia a un grupo de microorganismos denominados periodontopatógenos por los factores de virulencia que los caracterizan, entre ellos enzimas, toxinas y elementos estructurales que agreden los tejidos periodontales del huésped. Los más asociados son Porphyromona gingivalis, Tannerella forsytensis, Treponema dentícola y Aggregatibacter actinomycetemcomitans ( $\mathrm{Aa}$ ). El objetivo de este trabajo fue determinar la presencia de Aggregatibacter actinomycetemcomitans en individuos diagnosticados con enfermedad periodontal crónica. Se seleccionaron 55 pacientes y se tomaron muestras de placa subgingival con conos de papel absorbente. Se transportaron al laboratorio de Microbiología y fueron procesadas en medio TSVB. Se estudió la morfología colonial y la microscopía. En los 110 sitios estudiados no se obtuvo desarrollo del microorganismo; se aislaron colonias que se correspondían con morfología de cocobacilos Gram negativos característicos de Aa; podrían ser compatibles con Haemophilus aphrophilus, microorganismo común en la cavidad bucal $y$ en surcos gingivales.

\section{Palabras claves}

Periodontopatógenos, periodontitis, aislamiento de facultativos.

\section{Summary}

Periodontal disease is associated with a group of microorganisms by periodontopathogens 
called virulence factors that characterize including enzymes, toxins and structural elements that attack the periodontal tissue of the host. Most associates are Porphyromona gingivalis, Tannerella forsytensis, Treponema denticola and Aggregatibacter actinomycetemcomitans $(\mathrm{Aa})$. The aim of this study was to determine the presence of Aggregatibacter actinomycetemcomitans in individuals diagnosed with chronic periodontal disease. 55 patients were selected and subgingival plaque samples were collected with absorbent paper cones. Were transported to the microbiology laboratory and were processed in the midst TSVB. Colonial morphology was studied and microscopy. II 0 sites studied in no growth of the microorganism was obtained, colonies were isolated that corresponded to Gramnegative coccobacilli morphology characteristic of $\mathrm{Aa}$, they may be compatible with Haemophilus aphrophilus, microorganism common in the oral cavity and gingival sulci.

\section{Keywords}

Periodontopathogens, periodontitis, optional insulation.

\section{Resumo}

A doença periodontal está associada a um grupo de microrganismos por periodonto designados como factores de virulência que caracterizam incluindo enzimas, toxinas e os elementos estruturais que atacam 0 tecido periodontal do hospedeiro. A maioria dos associados são Porphyromona gingivalis, Tannerella forsytensis, Treponema denticola e Aggregatibacter actinomycetemcomitans (Aa. Oobjetivo deste estudo foi determinar a presença de Aggregatibacter actinomycetemcomitans em indivíduos com diagnóstico de doença periodontal crônica. 55 pacientes foram selecionados e amostras de placa bacteriana subgengival foram colhidas com cones de papel absorvente. Foram transportados para o laboratório de microbiologia e foram processados no TSVB meio. Morfologia colonial foi estudada e microscopia; I 10 locais estudados em nenhum crescimento do microorganismo foi obtida, as colónias foram isoladas que correspondeu a morfologia característica cocobacilos Gram- negativa de $\mathrm{Aa}$, podem ser compatíveis com Haemophilus aphrophilus microrganismo comum na cavidade oral e sulcos gengivais.

\section{Palavras chave}

Periodontopatógenos, periodontite, isolamento opcional.

\section{Introducción}

Las enfermedades gingivoperiodontales, afectan tanto el periodonto de protección como el de inserción; su origen está relacionado con el biofilm dental de ubicación subgingival formado por bacterias y sus productos. Actualmente a la presencia de biofilm se suman factores inherentes al propio huésped. La respuesta inmune del huésped tiende a la protección del mismo pero en ocasiones puede ocasionar daños que llevan a la destrucción de los tejidos'. Conceptos actuales permiten vislumbrar que la enfermedad periodontal pasa por períodos de actividad en los que pueden observarse pérdida ósea y otros en los que no. Muy frecuentemente el diagnóstico de la enfermedad periodontal es eminentemente clínico y radiográfico y orientan hacia el daño producido pero no alcanzan para establecer esos periodos de actividad. Para ello es necesario el respaldo del diagnóstico microbiológico e instaurar un tratamiento que lleve a la curación y no solo pasar a un periodo de inactividad. Desde un biofilm compatible con salud, integrado por Streptococos sp, Actinomyces sp, Veilonellas $s p$, Corynebactrerium sp y otros hasta llegar a una placa supragingival madura con la presencia de Haemophilus sp y treponemas. La sucesión autogénica complejiza la flora subgingival con un aumento de bacilos gram positivos y da lugar a una gingivitis (inflamación de la encía), la que puede $\circ$ no evolucionar a periodontitis cuando la presencia y proporción de microorganismos anaerobios estrictos aumenta; la mayoría de ellos son de forma bacilar y Gram negativos y se los considera colonizadores tardíos. A ellos se suman bacilos móviles y aumentan en número las formas espirilares. Las especies más asociadas a destrucción periodontal son Aggregatibacter (Actinobacillus) actinomycetemcomitans (Aa), Porphyromona gingivalis, Tannerella forsythensis (antes Bacteroides forsythus), Prevotella intermedia, Fusobacterium nucleatum, Campylobacter rectus y Eikenella corrodens'.

Aa es un bacilo o cocobacilo Gram negativo, que 
muchos autores consideran habitante comensal de la cavidad bucal principalmente el serotipo a. Slots et $\mathrm{al}^{2}$ en un estudio en el que comparó la presencia de $\mathrm{Aa}$ en lengua, mucosa yugal y bolsas periodontales halló a estos bacilos preferentemente en sacos periodontales. Fisiológicamente es capnófilo o anaerobio facultativo, inmóvil, no esporulado, oxidasa negativa, indol negativo, productor de catalasa. No requiere factores de crecimiento, fermenta carbohidratos específicos como el almidón, sacarosa, trehalosa y lactosa. Sus principales factores de virulencia son la producción de vesículas con toxinas extracelulares, la leucotoxina con actividad frente a los leucocitos y una toxina distensora de células epiteliales de la encía ${ }^{3}$. Su polisacárido O permite establecer además del serotipo a, los serotipos b, c, d, e, f y g; el serotipo b es considerado el más leucotóxico y está asociado a la periodontitis agresiva a pesar que algunos estudios proponen la presencia de este germen también en periodontitis crónicas ${ }^{4,5,6}$. No todos los serotipos tiene la misma actividad de virulencia sobre todo la basada en la acción de la leucotoxina.

$A a$ es un germen de requerimientos exigentes por lo tanto para su desarrollo en laboratorio existen varios medios de cultivo para su aislamiento. Algunos ${ }^{7}$ han propuesto medios enriquecidos como agar sangre o agar chocolate, suplementado con hemina, vitamina $\mathrm{K}$ y menadiona; Slots ${ }^{9}$ el medio TSBV (bacitracina,vancomicina) y Tzukubashi $^{10}$ el AAMM. El medio de Slots, contiene tripteína de soja, extracto de levadura, suero de caballo, bacitracina y vancomicina; estos antibióticos suprimen a la mayoría de las otras especies orales; a su vez permite un mayor aislamiento de Aa que los medios con sangre. Al ser un medio transparente permite observar macroscópicamente las colonias y distinguirla característicamente como una colonia de $0,5 \mathrm{a} / \mathrm{mm}$, transparente de bordes irregulares; presenta una formación estrellada en el centro y es adherente al agar, ambas características dadas por la gran adhesión que posee este microorganismo debido a sus fimbrias ${ }^{10}$. Algunas características fenotípicas permiten diferenciarlo de Aggregatibacter (antes Haemophilus) aphrophilus, también habitante de surcos gingivales y placas dentales. Este microorganismo, cocobacilo Gram negativo, anaerobio facultativo presenta colonias pequeñas (de 0,5 a $\mathrm{Imm}$ ) que necesitan más de $\mathbf{4 8}$ horas para obte- ner un gran desarrollo; son convexas, de aspecto granuloso y pigmento amarillento. Es catalasa y oxidasa negativo lo que permite diferenciarlo de $A a$, aunque es probable que existan cepas atípicas que presenten variabilidad en estas reacciones ${ }^{10}$. A diferencia de otros hemofilus no necesita de factores $X$ y $V$ para desarrollar y podrían por estudios de base molecular diferenciarse cepas atípicas de Aa de Haemophilus aphrophilus ${ }^{11,12}$. El objetivo de este estudio fue establecer la presencia de $A a$ en enfermedad periodontal por método de cultivo.

\section{Material y Método}

Participaron en este estudio 55 sujetos sistémicamente saludables que asistieron a las clínicas odontológicas de la Facultad de Odontología de la Universidad del Nordeste. Cada participante firmó el consentimiento informado aprobado por el Comité de Bioética de la institución. El estudio se realizó en pacientes con diagnóstico clínico de periodontitis crónica entre 35 y 65 años de edad. Los criterios de exclusión fueron embarazo, lactancia, presencia de diabetes o cualquier enfermedad sistémica que alterara el curso de la enfermedad periodontal, terapia periodontal en el último año y utilización de antimicrobianos en forma sistémica o tópica en los seis meses previos al examen clínico y a la toma de muestras microbiológicas. Después de realizar la historia clínica y el examen clínico y radiográfico completo, se registró la profundidad de bolsa con una sonda periodontal calibrada y se clasificó la enfermedad periodontal en leve (hasta $4 \mathrm{~mm}$ ), moderada (entre 4 y $6 \mathrm{~mm}$ ) y severa (más de 6 $\mathrm{mm}$ ).

Se seleccionaron por paciente dos sitios ( 110 en total), preferentemente el primer molar superior y el incisivo inferior, piezas en las que habitualmente comienzan las lesiones de periodontitis agresiva. Después de aislar la zona con algodón y remover la placa supragingival, se tomaron muestras del surco gingival con conos de papel absorbente $\mathrm{N}^{\circ} 30$ ó 35 durante 60 segundos (Fig. I), se transportaron en medio RTFI 3 hasta el laboratorio (Fig 2). A partir de las muestras subgingivales se hicieron cultivos microbiológicos en agar TSBV9 (tripteína soja, extracto de levadura, suero de caballo, vancomicina y bacitracina). Las cajas de agar TSBV se incubaron en una atmósfera 


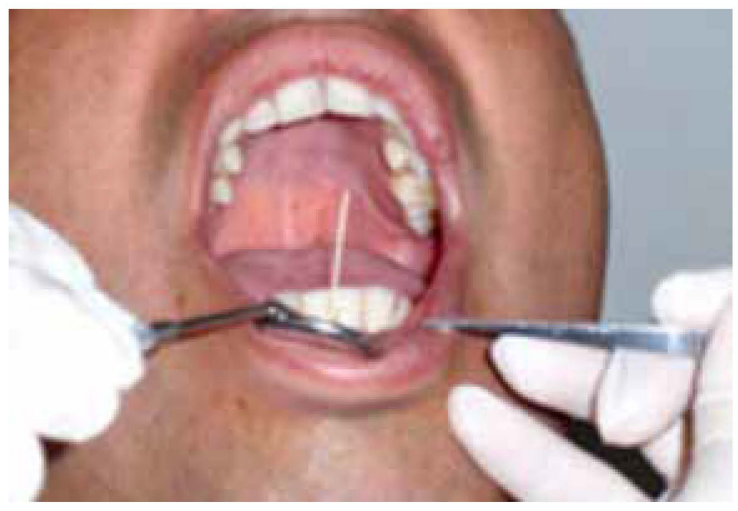

Fig. I. Toma de muestras.

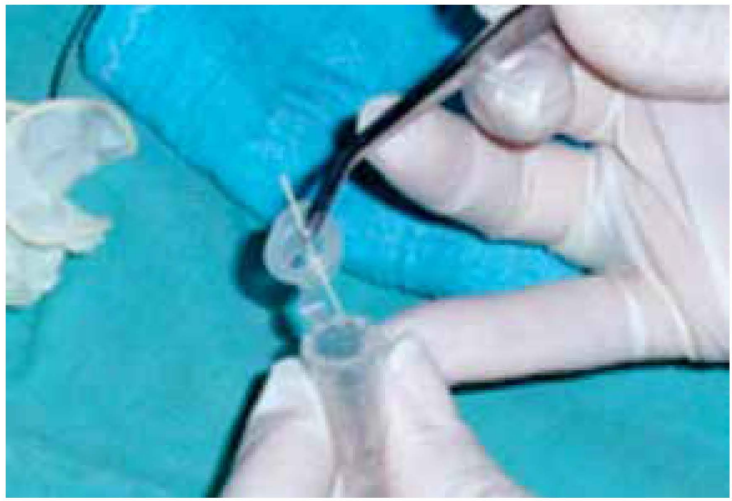

Fig. 2. Medio de transporte.

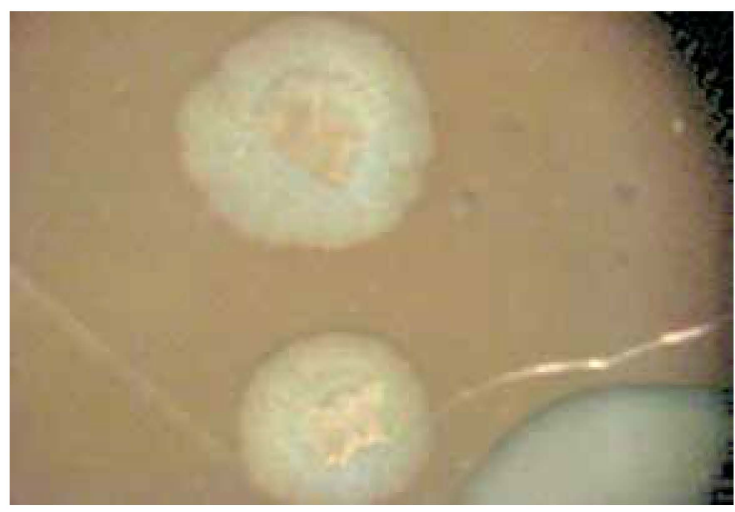

Fig. 3. Colonias transparentes con centro marrón (vista con lupa estereoscópica).

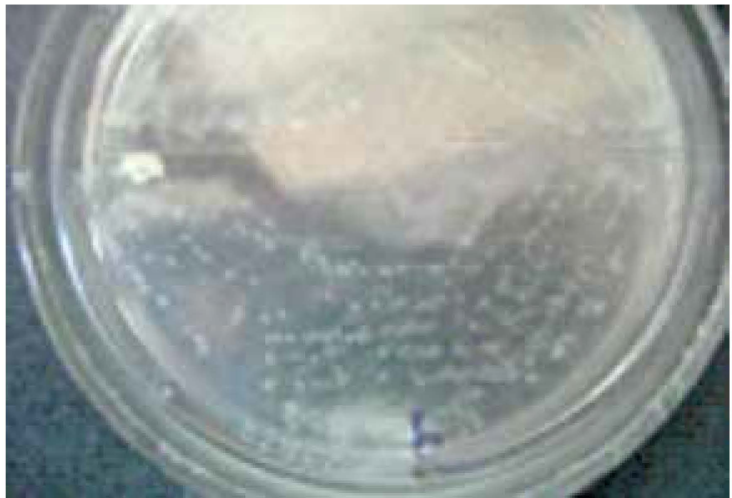

Fig. 4. Aislamiento de colonias transparentes con centro marrón.

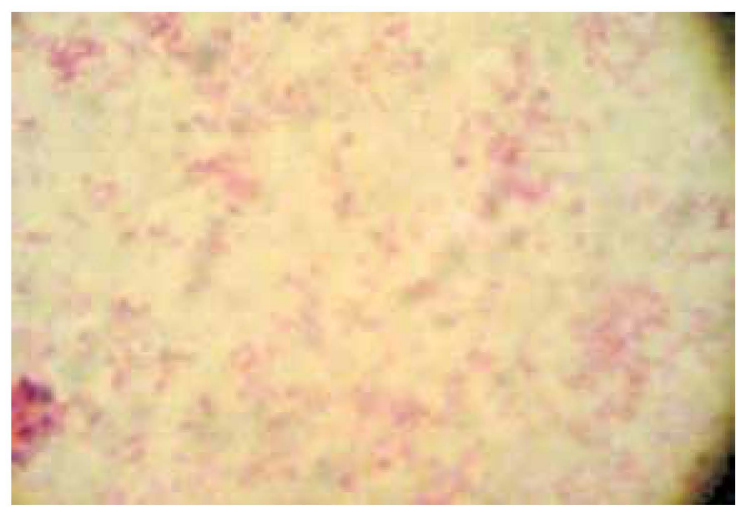

Fig. 5. Cocobacilos Gram negativos.

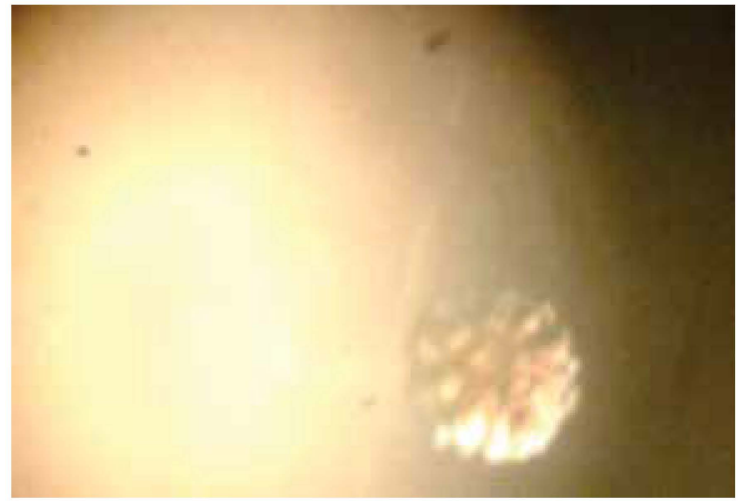

Fig. 6. Colonias rugosas, de borde irregular de 0,5 a Imm.

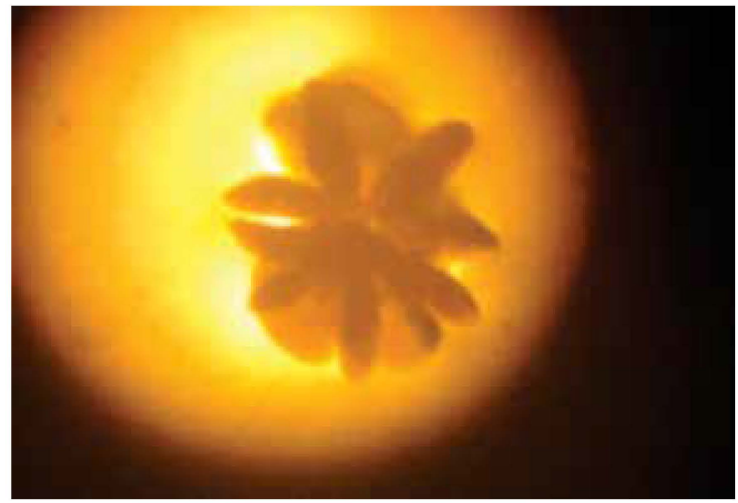

Fig. 7. Colonias de hasta $2 \mathrm{~mm}$, transparentes, de borde liso con estructuras similares a cigarros que forman un centro. 
al $5 \%$ de CO2 entre 3 y 5 días. La identificación se hizo teniendo en cuenta características morfológicas de las colonias, microscopía y pruebas bioquímicas adicionales.

\section{Resultados}

Los cultivos de las muestras de biofilm subgingival estudiadas permitieron aislar varias especies bacterianas entre las cuales se observaron diversas morfologías coloniales que se correspondieron con cocos positivos y negativos. Particularmente en un $20 \%$ de los sitios estudiados se obtuvieron colonias pequeñas de $0,5 \mathrm{~mm}$ a I y $2 \mathrm{~mm}$, transparentes de borde liso, con un centro más oscuro (figura 3 y 4 ) que correspondían a cocobacilos y cocos negativos (figura 5); cinco cepas presentaron reacción positiva a las pruebas de catalasa, oxidasa y glucosa. Estas cepas fueron obtenidas de surcos con bolsas de entre 7 y $8 \mathrm{~mm}$. En un 4,4\% se obtuvieron colonias de iguales características pero con borde irregular y de aspecto rugoso (fig.6) y también correspondieron a cocobacilos negativos; en un $7,7 \%$ se obtuvieron colonias de hasta $2 \mathrm{~mm}$, transparentes, de borde liso con estructuras similares a cigarros que forman un centro (fig. 7) y se correspondían con cocobacilos negativos; ambos tipos coloniales provenían de bolsas con profundidad de sondaje entre 4 y $6 \mathrm{~mm}$. Ninguna de las colonias presentó la forma de estrella que caracteriza a Aa según varios autores ${ }^{9,12}$.

\section{Discusión}

Varios autores han asociado la presencia de Aa con periodontitis crónicas y lo han comparado con la presencia de Porphyromona gingivalis y Tannerella fosythia hallando porcentajes menores de $\mathrm{Aa}^{14,15}$ que las especies anteriores. Ardila Medina et all 6 hallaron un 23\% de $\mathrm{Aa}$ en un estudios de periodontitis crónicas y en pacientes chilenos detectaron la presencia de $\mathrm{Aa}$ en un $25 \%$ de sitios activos y un $12,5 \%$ en sitios inactivos por lo que concluyen que no hay asociación entre $\mathrm{Aa}$ y periodontitis progresiva del adulto ${ }^{17}$. Utilizando biología molecular con PCR, Cortelli et al 4 halló una mayor relación de Aa con periodontitis severa, (23,13\%), con periodontitis moderada $17,42 \%$ y un $16,11 \%$ con periodontitis leves. Iguales resultados se obtuvieron 18 en un estudio multicéntrico realizado en pacientes colombianos, chilenos y españoles en los que la presencia de Aa fue menor en todos los grupos. En este trabajo hallamos que en sitios periodontales con profundidades de bolsa de más de 6 $\mathrm{mm}$, crecieron colonias transparentes de borde irregular y un centro más oscuro; cinco cepas presentaban forma de cocobacilos negativos $y$ respondieron positivamente a pruebas bioquímicas (catalasa, oxidase, glucosa) pero su aspecto colonial no presentaba la característica forma estrellada dentro de la colonia según la describe Olsen y colaboadores ${ }^{12}$; probablemente correspondían a serotipos que no presentan los factores de virulencia propios de destrucción periodontal, principalmente la presencia de fimbrias, responsable de la adhesividad entre bacterias y de la colonia al agar; deberían investigarse con biología molecular. El otro tipo colonial, por sus características fenotípicas, podría ser compatible con Aggegatibacter aphrophilus, coincidiendo con la habitual presencia de los mismos en cavidad bucal y en surcos gingivales. A. aphropilus, carece de potencial periodontopatógeno por la composición de su lipopolisacárido'2. Las colonias con estructuras centrales en forma de cigarro no podemos confirmar que pertenezcan a $\mathrm{Aa}$ como lo muestra Kesić et al en su trabajo.

\section{Conclusiones}

- En las muestras estudiadas no se logró el aislamiento de Aggregatibacter actinomycetemcomitans.

- Se hallaron colonias cuyas características macro y microscópicas se correspondían con cocobacilos negativos pero sólo un pequeño porcentaje respondió positivamente a las pruebas bioquímicas compatibles con $\mathrm{Aa}$. 
I. Bullón Fernández P. Diagnóstico por el laboratorio de las enfermedades periodontales y periimplantarias. Diagnóstico de la periodontitis. Av Periodon Implantol. 2004; I6, I: 35-45.

2. Slots J, Reynolds $\mathrm{H}$ and Genco R. Actinobacillus actinomycetemcomitans in Human Periodontal Disease: a Cross-Sectional Microbiological Investigation Infect Immun. 1980 sep; 29 (3): 1013-1020.

3. Díaz Zúñiga J, Yáñez Figueroa J, Melgar Rodríguez S, Álvarez Rivas C, Rojas Lagos C, Vernal Astudillo R. Virulencia y variabilidad de Porphyromonas gingivalis y Aggregatibacter actinomycetemcomitans y su asociación a la periodontitis Rev Clin Periodoncia Implantol Rehabil Oral 2012. Vol. 5 (I); 40-5.

4. Cortelli JR, Roman-Torres CVG, Aquino DR, Franco GCN, Costa FO, Cortelli SC. Occurrence of Aggregatibacter actinomycetemcomitans in Brazilians with chronic periodontitis. Braz Oral Res. 2010 Apr-Jun; 24 (2): 217-23.

5. Armitage GC. Comparison of the Microbiological features of Chronic and aggressive Periondontitis. Periodontol 2000. 2010; 53: 70-88.

6. Jardim Junior EG, Bosco JMD, Lopes AM, Landucci LF, Jardim ECG, Carneiro SRS. Occurrence of Actinobacillus actinomycetemcomitans in patients with Crhonic Periodontitis, Aggressive Periodontitis, Healthy subjects and children with Gingivitis in two cities of the state of Sao Paulo, Brazil. J appl Oral Sci. 2006; I4 (3): I53-I56.

7. Kesić L, Petrović M, Obradović R, Pejčić A. The importance of Aggregatibacter actinomycetemcomitans in etiology of periodontal disease - mini review Acta Medica Medianae 2009; 48 (3): 36-37.

8. Van Steenbergen TJM, Van Winkelhoff AJ, Vander Mispel L, Vander Velden U, Abbas F, De Graaff J, Comparison of two selective media for Actinobacillus actinomycetemcomitans. J Clin Microbiol. 1986; 24 (4): 636-638.

9. Slots J. Selective medium for isolation of Actinobacillus actinomycetemcomitans. J Clin Microbiol 1982 April; 15 (4): 606-609.

10. Tsuzukibashi O, Takada K, Saito M, Kimura C, Yoshikawa T, Makimura M, Hirasawa M. A novel selective medium for isolation of Aggregatibacter (Actinobacillus) actinomycetemcomitans. J Periodontal Res. 2008 Oct; 43 (5): 544-8.

II. Win W, Allen S, Janda W, Koneman E, Procop G, Schereckenberger P, Woods G. Koneman. Diagnóstico microbiológico. Texto y atlas en color. $6^{\circ}$ Panamericana. 2008

12. Olsen I, Shah A, Gharbia S. Taxonomv and bio- chemical characterhtics of Actinobacillus actinomycetemcomitans and Porphyromonas gingivalis. Periodontology 2000, 1999; 20: 14-52.

13. Van Steenbergen TJM, Petit MDA, Tijhof CJ, Van Winkelhoff AJ, Vander Velden V, De Graaff J. Survival in transport media of Actinobacillus actinomycetemcomitans, Porphyromonas gingivalis and Prevotella intermedia in Human subgingival samples. Oral Microbiol Immunol. 1993; 8: 370-374.

14. Rylev M,Kilian M.Prevalence and distribution of principal periodontal pathogens worldwide. J Clin Periodontol 2008; 35:346-36I

15. Mayorga-Fayad I, Lafaurie Gl, Contreras A, Castillo DM, Barón A, Aya MR. Subgingival microbiota in chronic and aggressive periodontitis in Bogotá, Colombian: epidemiological approach. Biomedica 2007; 27: 21-33.

16. Ardila Medina CM, Alzate Vega J, Guzmán Zuluaga C. Association of Aggregatibacter actinomycetemcomitans and red complex microorganisms with clinical parameters of patients with chronic periodontitis. AMC 2010; 14, 3 Mayo-jun. 2010 versión On-line ISSN 1025-0255.

17. López N. Occurrence of Actinobacillus actinomycetemcomitans, Porphyromonas gingivalis, and Prevotella intermedia in Progressive Adult Periodontitis J Periodontol 2000; 7I: 948-954.

18. Herrera D, Contreras A, Gamonal J, Oteo A, Jaramillo A, Silva N, Sanz M, Botero J, León R. Subgingival microbial profiles in chronic periodontitis patients from Chile, Colombia and Spain J Clin Periodontol 2008; 35: 106-I I 3. 